\title{
Implementing electronic medical records in Canadian emergency departments
}

\author{
Daniel Rosenfield, B.Arts.Sc, MD, MHI(c)*; Gregory Harvey, MD*; Karim Jessa, MD* ${ }^{\dagger}$
}

\section{INTRODUCTION}

The movement toward electronic medical records (EMR) continues to gain traction across the Canadian landscape. The emergency department (ED) is a complicated place, with a myriad of disparate workflows, patient requirements, and safety considerations. As such, careful consideration is necessitated before "going live" with any new or upgraded EMR, as the ramifications of rushing or poorly piloting new systems would have significant patient safety and efficiency implications. In this paper, we discuss considerations, both technologic and human, that must be adequately examined before, during, and after the implementation of an EMR system in the ED.

\section{PREPARATION}

Implementing a new EMR is a significant undertaking, requiring support from leadership and buy in from front-line staff. A hospital-wide organizational framework with specific ED liaisons is essential, as they will guide overarching principles. ${ }^{1}$

Identifying and engaging physician, nursing, and clerical champions is essential. ${ }^{2}$ Champions can help the project team engage front-line clinicians directly, point out unseen deficiencies, and act as the "devil's advocate" for any given proposed change. Engaging patients during the planning stages is important, as they can offer valuable insights not visible to those working from the "inside." Additionally, offering dedicated time and funding to all staff who would be affected by the change, to aid in the development, testing, and training on the new system, would result in greater engagement. Assessing change readiness to decide what kind of implementation (gradual/phased v. comprehensive/"big bang") should be selected would help facilitate the best adoption strategy. ${ }^{3-5}$ For example, if a particular ED or hospital is entirely paper based, a big bang approach may be less desirable than one employing gradual implementation.

The implementation of a new EMR in the ED offers a natural opportunity to examine current and idealized workflows (i.e., processes). The EMR should not wholly change organizational culture or practices; however, clear prioritization of patient outcomes and critical efficiency changes is necessary. If workflow modification is desired, engaging the appropriate stakeholders is essential. For example, if lessons learned from morbidity and mortality rounds can be incorporated with a new EMR, this should be a priority. Hospital-specific and provincially mandated key performance indicators should be actively considered during the design, build, and implementation of the EMR. For example, new electronic tools should be designed to minimize the patient length of stay and provider initial assessment time. Attention is also required during design and build sessions, as decisions made in the ED can have adverse workflow consequences for the rest of the hospital and vice versa, for example, with the use of "hard stops" or "pop-ups/alerts."

One major advantage of EMRs over that of paper is the prospect of easily available data that can be queried, presented, and displayed. However, prior to implementation, each hospital must decide on a data utilization framework, as data are only useful if inputted correctly and consistently. Many issues must be considered, including which fields are mandatory (i.e., hard stops) and which fields require specific selections v. free text. If these choices are not thought out in advance, they can be nearly impossible to change after implementation.

From the *Division of Pediatric Emergency Medicine, Hospital for Sick Children, University of Toronto, Toronto, ON; and tInformation Management Technology (IMT) Department, Hospital for Sick Children, University of Toronto, Toronto, ON.

Correspondence to: Dr. Daniel Rosenfield; University of Toronto, Pediatric Emergency Medicine, Hospital for Sick Children, 555 University Avenue, Suite 1201, Toronto, ON, M5G 2L3; Email: daniel.rosenfield@sickkids.ca 
Implementation teams must focus on preserving provider efficiency that can be compromised by EMR adoption. ${ }^{6,7}$ Leadership should employ multiple risk mitigation strategies including resourcing sufficient workstations; using dictation devices, templates, and macros within note elements; creating order sets/ laboratory panels; and consolidating referral processes. Additionally, making pre-go-live training and post-golive optimization tips readily available would improve end-user engagement. Indeed, when implementing these techniques, some EDs have had no changes in efficiency when going live with EMRs. ${ }^{8,9}$

Communication with the organization and front-line providers throughout the preparation process and for months prior to going live is essential. Strategies include a combination of online communiques via email, intranet posting, live signage within the hospital, and social media (Twitter, Facebook, and Instagram) updates. ${ }^{10}$ These messages should come from the chief executive officer (CEO), chief medical information officer (CMIO), division heads, and local implementation teams, respectively, each highlighting specific goals relevant to the individual leader's portfolio. The most important communication is face to face; thus, dedicating effort to engaging those affected by the change directly is the best use of communication resources. Clinicians should not be surprised about the upcoming go live, nor should they be seeing the interface for the first time when they are being trained. Leadership should also solicit input from front-line staff and provide monthly meeting opportunities for them to see and trial the interface well in advance of going live.

During end-user training, sessions must focus on workflows. These sessions should ideally involve relatively small groups to encourage engagement and a "specialist training specialist" model in which peers with additional training teach their colleagues to improve provider buy in. ${ }^{10}$ These sessions often represent the first time the EMR change is "real" for many and is an opportunity to raise questions and concerns. A hypothetical benefit of having a colleague provide training is decreased hostility from endusers who are facing a change in workflows.

\section{GO LIVE}

Since most EDs cannot temporarily close, extensive organizational and vendor support is required to assure patient safety and a successful launch. Prior to go live, a clear "cutover" strategy must be developed, to identify how to assure no data or patients are "lost" in the changeover and to troubleshoot disparate workflows. Clinicians also need access to legacy chart data.

Go live is typically a particular time point but can be over a number of days to weeks, depending on the type of implementation. At go live, "at the elbow" support is the most common way vendors and hospitals can help ED staff. Specifically, individual "super users" of the software must be available in the ED 24/7 in sufficient quantity such that they can answer any questions and support all front-line staff. Having as many local champions who work in the department who are known to staff is ideal, but the department must also have a complement of easily identifiable (using either a specially coloured shirt or buttons) external super users. Additionally, scheduling go live during the time of year with the lowest ED visits and fewest boarders is ideal. Leadership should plan for increased staffing and expectations of decreased efficiency during the immediate go-live period. If go live is in the summer months, leadership should use a winter staffing schedule. These additional clinicians should be added for at least one to two months following go live, as many departments do not regain their historical efficiency until that time (at the earliest). ${ }^{6}$ Finally, it is advisable to put clinicians who are comfortable with the system on shift during go live if possible.

At the hospital level, leadership should engage staff with morale-boosting techniques such as free lunch/coffee as an acknowledgement of the additional stress on front-line staff. Posted notices in the waiting room, as well as locally and on social media, can also be used to advise patients of potentially longer ED wait times. Depending on a contained (i.e., ED only) or hospital-wide implementation, these logistical concerns may be exacerbated. If a go live is hospital wide, cross-training ED super users to support consulting services would help facilitate ED and inpatient workflows and minimize disruptions for ED clinicians.

\section{POST-GO-LIVE OPTIMIZATION}

Following a "stabilization" phase in which the initial bugs and workflows are corrected, a dedicated "optimization" phase is essential to implement many of the ideas that were deemed non-essential during preparation and go live. ${ }^{11} \mathrm{~A}$ governance and prioritization framework is crucial to use available support resources efficiently. Attention to safety measures and critical incident tracking can help in decision making. These include individual clinician-specific projects, quality 


Table 1. Reportable metrics available from EMRs
\begin{tabular}{ll}
\hline Clinicians/division leadership & Admission rate \\
& Investigation rate (e.g., average number of CBCs ordered) \\
& Readmission rate (within 72 hours, i.e., bounce back) \\
& Individual and group time parameters (physician initial assessment time, length of stay, etc.) \\
& EMR efficiency (i.e., mouse clicks/time in EMR per patient) \\
Hospital leadership & Medication reconciliation \\
& Percentage of orders entered electronically \\
& Number of medications administered through barcode scanning Overtime tracking \\
\hline $\mathrm{CBC}=$ complete blood count; EMR=electronic medical record.
\end{tabular}

improvement initiatives, and workflow optimization. During this time, utilization of the original project team (with protected time), along with frequent communication with front-line clinicians, must be maintained. Once optimization requests are prioritized, they can be evaluated using a quality improvement framework, as making EMR modifications are aptly suited to the plando-study-act cycles highlighted in quality improvement (QI) literature. ${ }^{12}$ As most EMRs offer a cornucopia of metrics, leadership must diligently examine which are desired. Evaluation of successes and failures must be ongoing, using metrics relevant to individual clinicians, hospital leadership, and local health authorities. Some common examples are included in Table 1. Reports should be generated based on a priori defined parameters, which are often specific to local institutions or provinces. For example, in Ontario, pay-for-results funding schemes will incentivize the minimization of provider initial assessment times; therefore, the use of EMRs to trend these times is essential.

\section{CONCLUSION}

Aside from physically moving an ED or hospital, EMR integration is one of the biggest changes to affect both clinician and patient experiences. If done correctly, modern EMRs offer a significant opportunity to improve efficiency, communication, and patient safety. They can also enhance the patient experience by facilitating better discharge instructions and appointment and test follow-up. However, poor implementation can cause staff attrition and inefficient ad hoc workarounds. As more Canadian EDs and hospitals move toward or upgrade their electronic systems, these lessons will need to be increasingly heeded. Organizations should share evidence-based implementation strategies, and hospital networks should strive for the long-term goal of a fully functional system that presents data clearly, enhances patient safety, and improves the provider experience.

Keywords: Electronic medical record, emergency department implementation

\section{REFERENCES}

1. Appelbaum SH, Habashy S, Malo JL, Shafiq H. Back to the future: revisiting Kotter's 1996 change model. 7 Manage Dev 2012;31(8):764-82.

2. Melnyk BM. Culture eats strategy every time: what works in building and sustaining an evidence-based practice culture in healthcare systems. Worldviews Evid Based Nurs 2016;13 (2):99-101.

3. Hung D, Chen PH. Ready for change? The role of physician and staff engagement, burnout, and workplace attributes. 7 Ambul Care Manage 2017;40(2):150-7.

4. Kulhanek BJ. Creating effective electronic medical record change management processes. Comput Inform Nurs 2011;29 (8):431-5.

5. Mustain JM, Lowry LW, Wilhoit KW. Change readiness assessment for conversion to electronic medical records. $\mathcal{Z}$ Nurs Adm 2008;38(9):379-85.

6. Feblowitz J, Takhar SS, Ward MJ, Ribeira R, Landman AB. A custom-developed emergency department provider electronic documentation system reduces operational efficiency. Ann Emerg Med 2017;70(5):674-682.e1.

7. Spellman Kennebeck S, Timm N, Farrell MK, Spooner SA. Impact of electronic health record implementation on patient flow metrics in a pediatric emergency department. $\mathcal{F}$ Am Med Inform Assoc 2012;19(3):443-7.

8. Hill RG Jr, Sears LM, Melanson SW. 4000 clicks: a productivity analysis of electronic medical records in a community hospital ED. Am 7 Emerg Med 2013;31(11):1591-4.

9. Tall JM, Hurd M, Gifford T. Minimal impact of an electronic medical records system. Am 7 Emerg Med 2015;33 (5):663-6.

10. McCarthy C, Eastman D. Change Management Strategies for an Effective EMR Implementation. Chicago: HIMSS.

11. Steele R, Adkins W. EHR Go-Live. HIMSS; 2014.

12. Institute for Healthcare Improvement IHI. Plan-Do-StudyAct (PDSA). Cambridge, MA. Available at: http://www.ihi. org/resources/Pages/Tools/PlanDoStudyActWorksheet.aspx (accessed August 1, 2018). 